\title{
Optically Controlled Reconfigurable Antenna Array for mm-Wave Applications
}

\author{
I. F. da Costa, Arismar Cerqueira S., Jr., D. H. Spadoti, L. G. da Silva, \\ J. A. J. Ribeiro, and S. E. Barbin
}

\begin{abstract}
This letter reports the concept and development of the first optically controlled reconfigurable antenna for millimeterwave (mm-wave) applications. It is based on a slotted-waveguide antenna array and two photoconductive switches, which are used to control the slot electrical length. This novel photonics-based reconfigurable strategy enables frequency tunability and radiation pattern reconfiguration through the 28 - and $38-\mathrm{GHz}$ frequency bands. Numerical simulations and experimental results illustrate its applicability for mm-wave indoor applications. The measured gain is approximately 8.0 and $9.0 \mathrm{dBi}$ at 28 and $38 \mathrm{GHz}$, respectively.
\end{abstract}

Index Terms-Antenna arrays, millimeter-wave (mm-wave), optically controlled antennas, reconfigurable antennas.

\section{INTRODUCTION}

$\mathbf{R}$ ECONFIGURABLE antennas have attracted the attention of researchers due to their flexibility and potential properties for the next generations of wireless networks [1]. They allow reconfiguring not only the antenna frequency resonance, but also its bandwidth, radiation pattern, and polarization [1]-[3]. There are basically four reconfigurable techniques [3]: mechanical reconfiguration, electrical reconfiguration using $\mathrm{p}$-i-n diodes, optical reconfiguration, and structures built with liquid crystal.

Most of the reconfigurable antennas reported in the literature have been developed using electrical reconfiguration or microelectromechanical systems. These classical mechanisms have the drawback of adding complex system requirements to modify their radiating structure [3]. Optically controlled reconfigurable antennas provide unique advantages when compared to those classical approaches, such as easy integration into optical systems and absence of bias lines, which imply in the elimination

Manuscript received January 7, 2017; revised March 30, 2017; accepted April 25, 2017. Date of publication May 2, 2017; date of current version July 31, 2017. This work was supported in part by Finep/Funttel Grant 01.14.0231.00, under the Radio Communications Reference Center (CRR), in part by CNPq, CAPES, MCTI, and FAPEMIG, and in part by technical support from Anritsu, Keysight, and ESSS-ANSYS. (Corresponding author: Arismar Cerqueira Sodré Junior.)

I. F. da Costa and D. H. Spadoti are with the Federal University of Itajubá, Itajubá 37500-903，Brazil (e-mail: igorfelicianocosta@gmail.com; spadoti@unifei.edu.br).

A. Cerqueira Sodré Jr., L. G. da Silva, and J. A. J. Ribeiro are with the Laboratory WOCA (Wireless and Optical Convergent Access), National Institute of Telecommunications, Santa Rita do Sapucaí 37540-000, Brazil (e-mail: arismar@inatel.br; luis.gustavo@inatel.br; justino@inatel.br).

S. E. Barbin is with the State University of São Paulo, São Paulo 05508-900, Brazil (e-mail: barbin@usp.br).

Color versions of one or more of the figures in this letter are available online at http://ieeexplore.ieee.org.

Digital Object Identifier 10.1109/LAWP.2017.2700284 of unwanted interference, losses, and radiation pattern distortion [1]-[3]. The optical reconfiguration operational principle is based on the use of photoconductive switches, made of a semiconductor material (silicon or gallium arsenide) [4]-[7].

This letter proposes the concept and development of the first optically controlled reconfigurable antenna array (OCRAA), operating in the lightly licensed 28 - and $38-\mathrm{GHz}$ frequency bands for millimeter-wave (mm-wave) applications. The use of photoconductive switches allows to optically control its operational bandwidths and radiation pattern. The antenna presents a $90^{\circ}$ half-power beamwidth in the azimuth plane, with the purpose of enabling the coverage in an indoor environment.

The remainder of the manuscript is organized as follows. Section II presents the design of a multiband slotted-waveguide antenna array (SWAA). Section III reports the development of the optically controlled reconfigurable antenna array, whereas Section IV presents its numerical and experimental results. Finally, conclusions are addressed in Section V.

\section{MultiBAnd SWAA}

A SWAA presents the advantage of having the radiating elements integrated into its structure. This convenient arrangement negates the need for baluns or impedance-matching network structures, simplifying the antenna design. As its name suggests, a SWAA consists of lengths of waveguide with slots milled into its conducting walls. These slots introduce discontinuities in the conductor and, thus, they interrupt the flow of current along the waveguide. The slots act as dipole antennas since the current flows around their edges. SWAAs provide the following advantages [8]-[9]: compact and rugged construction, low loss, high power handling, and linear polarization.

SWAAs are typically based on the design procedures published by Elliot and Kurtz [10]. The slot length, interslot distance, and space between the last slot and structure end are approximately one-half of the guided wavelength. The bandwidth of such arrays is determined by the waveguide structure and resonant properties of the individual slots. SWAAs provide high gain in order to overcome free-space losses, which are natural for the mm-wave frequency range. However, conventional SWAAs are typically narrow and single-band [11].

We have previously developed a multiband SWAA using a novel structure with two different slot lengths placed on the waveguide narrowest face [9]. Our similar antenna array, here optically controlled, has the dimensions presented in Fig. 1. 


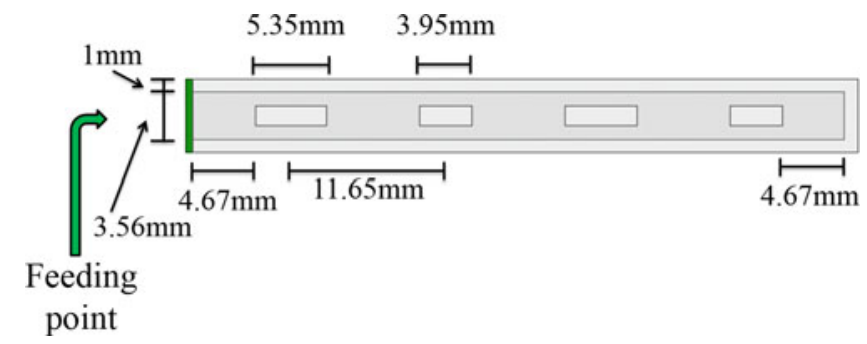

Fig. 1. Multiband SWAA design based on two different slot lengths.

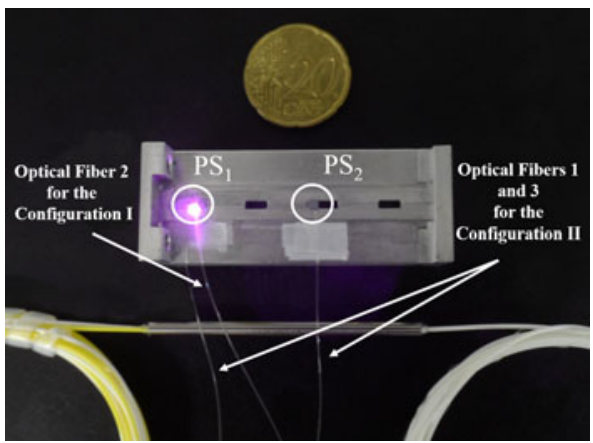

Fig. 2. Photograph of the optically controlled reconfigurable SWAA.

It consists of four slots with two different lengths, 5.35 and $3.95 \mathrm{~mm}$, which are shorter than one half of the guided wavelength (8.14 and $4.75 \mathrm{~mm}$ for 28 and $38 \mathrm{GHz}$, respectively), as in conventional SWAAs. We needed to optimize the slot lengths and all other dimensions using HFSS because of the mutual impedance between the slots with different lengths and, also, due to the influence of the photoconductive switch conductivity and permittivity for the ON/OFF-states, which are going to be explained in Section III.

\section{OPticAlly CONTROLLED FREQUENCY-RECONFIGURABLE SLOTTED WAVEGUIDE ANTENNA ARRAY}

This section presents the development of the optically controlled frequency-reconfigurable antenna for the mm-wave range, which is based on the multiband SWAA presented in Section II. Two photoconductive switches have been integrated to its structure in order to make it reconfigurable in the optical domain.

The OCRAA is presented in Fig. 2. It uses an aluminum waveguide milled with slots and consists of the following parts: two intrinsic silicon photoconductive switches with $0.3 \mathrm{~mm}$ thickness, two 808-nm lasers, one half-power optical splitter, and three standard single-mode optical fibers. The switches are soldered to small pieces of copper tape that have been previously glued to the waveguide body for improving their fixation quality. The lengths of the photoconductive switch $1\left(\mathrm{PS}_{1}\right)$ and photoconductive switch $2\left(\mathrm{PS}_{2}\right)$ are 5.5 and $1.4 \mathrm{~mm}$, respectively. $\mathrm{PS}_{2}$ had been appropriately designed to correspond to the difference between the slots' lengths. Finally, we have slightly inclined the optical fibers by using thicker pieces of copper tape to illuminate the switches from the top. It is one of the requirements to implement the optical control by using photoconductive switches.

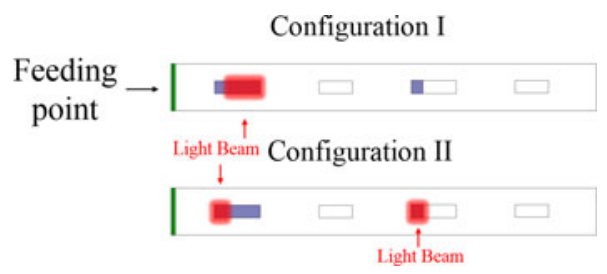

Fig. 3. Two configurations of the optically controlled reconfigurable SWAA.

The switch operation regime is "ON/OFF-states": "ON-state" when the silicon die is illuminated by a laser beam; "OFF-state" if no light is coming onto the switches. As soon as a switch is illuminated by light from the top, silicon changes from an insulator to a near-conducting state due to the creation of electron-hole pairs [12]. Light in the near-infrared region is adequate for this process because it strikes a balance between the absorption coefficient and light penetration depth. In order to experimentally demonstrate this effect and properly characterize our photoconductive switches, we have measured their conductivity for both states. The obtained value for the "OFF-state" was $437 \mu \mathrm{S} / \mathrm{m}$, which is in agreement with [13]. The PS conductivity significantly increases to $23.5 \mathrm{mS} / \mathrm{m}$ by illuminating it with the $808-\mathrm{nm}$ laser. Therefore, it is possible to manage the switch conductivity by controlling the optical incident power [4]. The photoconductive switch has been further experimentally characterized by measuring its rise and fall times, which are approximately 19 and $33 \mu \mathrm{s}$, respectively.

Most optically controlled reconfigurable antennas reported in the literature [4]-[7] are based on printed antennas, in which the photoconductive switches are in the direct path of the antenna surface currents, so losses in the PSs have a direct impact on the antenna efficiency. On the other hand, our novel approach relies on the use of PSs for managing the slot electrical length and, consequently, the antenna resonance, by means of suitably blocking part of the energy that would be radiated to the air. Therefore, the insertion loss is negligible.

The use of two switches on two different slots enables to provide different configurations for the antenna frequency response, as presented in Fig. 3. The first slot might be strategically covered using $\mathrm{PS}_{1}$, whereas the third slot can be partially covered using $\mathrm{PS}_{2}$. By entirely or partially closing a slot, one can manipulate its electrical length and, consequently, reconfigure the antenna bandwidth and radiation pattern. Our strategy allows reconfigurability through the $28-$ and $38-\mathrm{GHz}$ frequency bands, using two different configurations, namely:

1) Configuration I: $\mathrm{PS}_{1}$ on "ON-state," illuminated by the optical fiber $2\left(\mathrm{OF}_{2}\right)$, in order to cover the right part of slot 1 and $\mathrm{PS}_{2}$ on "OFF-state." This configuration enables the antenna operation in the $28-\mathrm{GHz}$ frequency band.

2) Configuration II: $\mathrm{PS}_{1}$ on "ON-state," illuminated by the optical fiber $1\left(\mathrm{OF}_{1}\right)$, for covering the left part of slot 1 and $\mathrm{PS}_{2}$ on "ON-state," illuminated by the optical fiber 3 $\left(\mathrm{OF}_{3}\right)$ to cover the left part of slot 2 . This configuration results in the antenna operating in the $38-\mathrm{GHz}$ frequency band. 

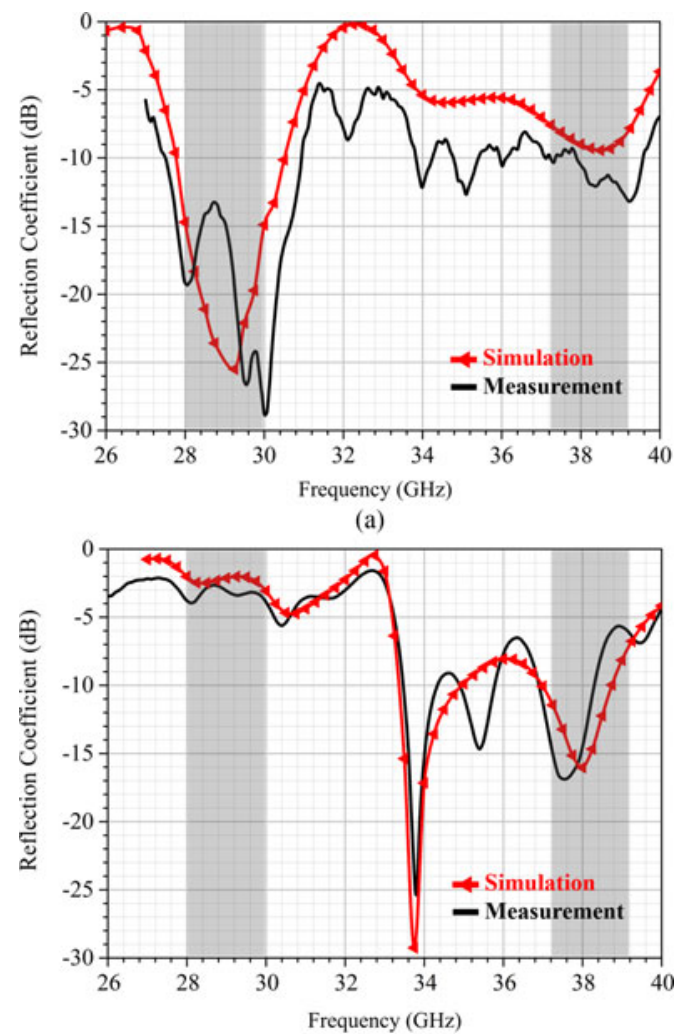

(b)

Fig. 4. OCRAA reconfigurable frequency response. (a) Configuration I. (b) Configuration II

\section{NUMERICAL AND EXPERIMENTAL RESULTS}

Three different experiments have been carried out in an indoor environment, with the purpose of evaluating the OCRAA performance in a realistic application scenario, namely: reflection coefficient measurement $\left(S_{11}\right)$, radiation pattern measurement, gain analysis as a function of frequency for the two proposed configurations. The OCRAA frequency response is presented in Fig. 4, which reports HFSS numerical simulations and experimental results of $S_{11}$ parameter for the "ON-state" and "OFF-state" of $\mathrm{PS}_{1}$ and $\mathrm{PS}_{2}$. The antenna numerical model takes into account the variation of the PS conductivity and permittivity as follows: Its conductivity has been defined in accordance to its characterization, presented in Section III, i.e., $437 \mu \mathrm{S} / \mathrm{m}$ and $23.5 \mathrm{mS} / \mathrm{m}$ for the "OFF-state" and "ON-state," respectively; whereas its permittivity has been set as 11.9 (standard value for the intrinsic silicon) and 10.3 for the "OFF-state" and "ON-state," respectively. The considered permittivity values are similar to those reported by Yadav et al. [14]. The simulated and experimental results are in good agreement for both configurations. Fig. 4(a) presents the result for the $\mathrm{PS}_{1}$ on "ONstate," in which the OCRAA operates in the 28-GHz frequency band from 27.6 to $30.8 \mathrm{GHz}$. Additionally, for $\mathrm{PS}_{1}$ and $\mathrm{PS}_{2}$ on "ON-state," its bandwidth is reconfigured to $38-\mathrm{GHz}$ band, from 36.8 to $38.4 \mathrm{GHz}$, as demonstrated in Fig. 4(b). The feeding structure excites the waveguide's entire operational frequency range, whereas the slots select the SWAA bandwidth based on their electrical lengths that correspond to approximately one

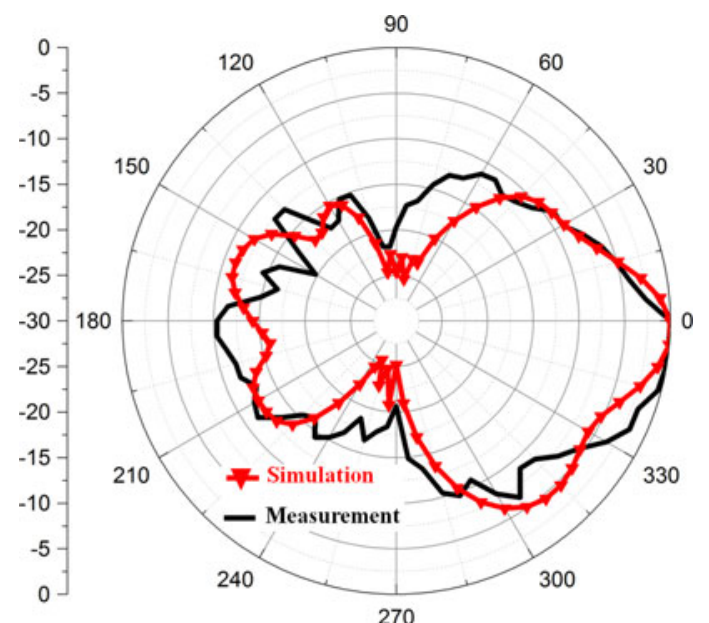

(a)

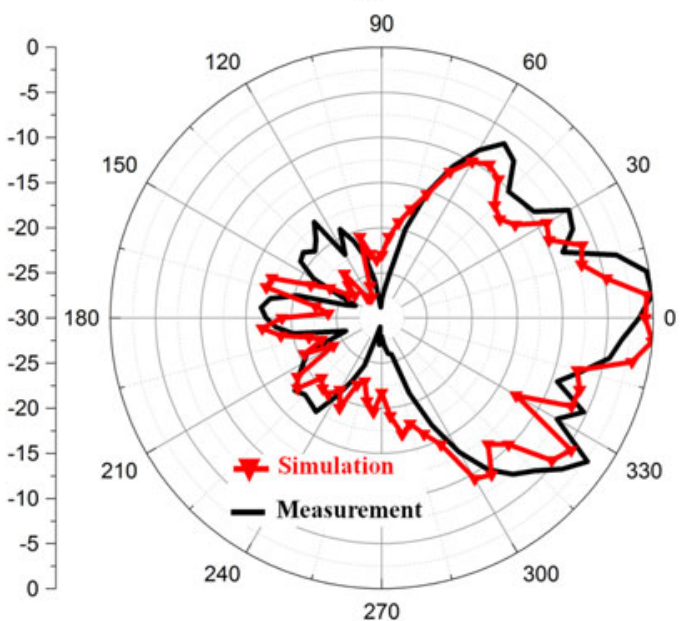

(b)

Fig. 5. OCRAA radiation pattern in the azimuth plane. (a) $28 \mathrm{GHz}$ (b) $38 \mathrm{GHz}$.

half of the guided wavelength for 28 and $38 \mathrm{GHz}$. Additional resonances at 34 and $35.4 \mathrm{GHz}$ are also observed due to the mutual coupling among different slots.

The next step was the OCRAA radiation pattern characterization. Fig. 5 displays a comparison between its numerical simulations and measurements, which are in good agreement for both frequency bands. The OCRAA gain is approximately 8.0 and $9.0 \mathrm{dBi}$ at 28 and $38 \mathrm{GHz}$, respectively. As expected, the antenna gain for the upper frequency band is higher since its area in terms of wavelength is larger. In both bands, the proposed OCRAA provides a directional radiation pattern, with the purpose of enabling coverage in an indoor environment.

The last experiment relies on a performance investigation based on a gain analysis as a function of frequency and optical reconfigurability. Its main goal is demonstrating the proposed approach applicability in reconfigurable 5G optical-wireless networks. Four different scenarios have been implemented for both frequency bands:

1) Scenario $1(S c 1)$ : OCRAA at the configurations I and II for 28 and $38 \mathrm{GHz}$, respectively; 


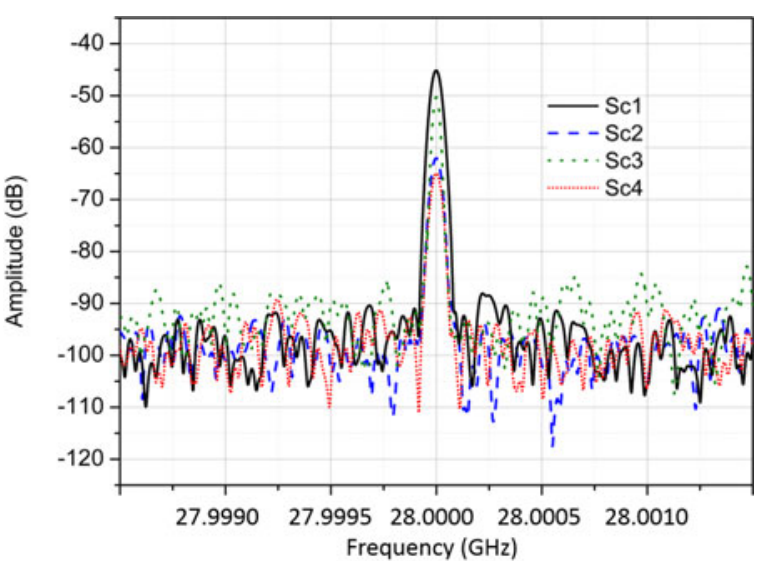

(a)

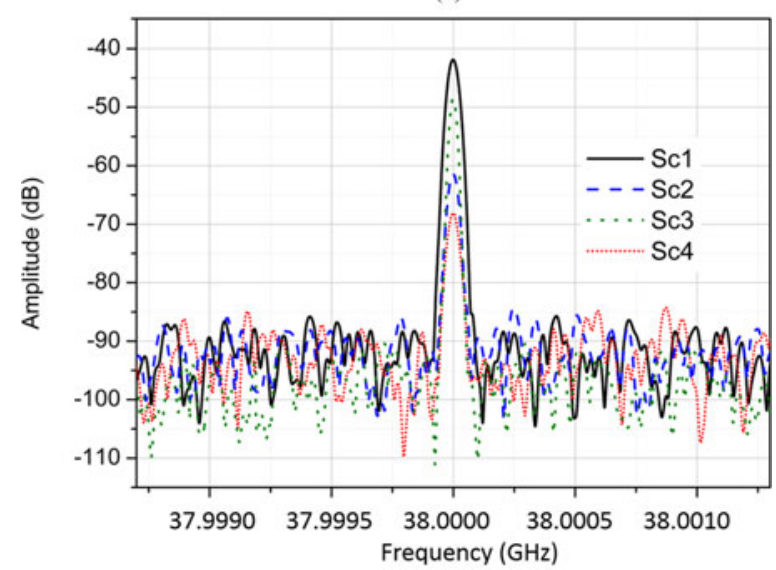

(b)

Fig. 6. Measured spectra for the four different scenarios. (a) $28 \mathrm{GHz}$. (b) $38 \mathrm{GHz}$.

2) Scenario 2 (Sc2): OCRAA with $\mathrm{PS}_{1}$ and $\mathrm{PS}_{2}$ on "OFFstate," keeping the same alignment of $\mathrm{Sc1}$;

3) Scenario 3 (Sc3): OCRAA with $\mathrm{PS}_{1}$ and $\mathrm{PS}_{2}$ on "OFFstate" and a new antenna alignment;

4) Scenario 4 (Sc4): SWAA without any PS.

The proposed OCRAA has been used to transmit an RF carrier, generated by an EXG signal generator from Keysight at $0 \mathrm{~dB} \cdot \mathrm{m}$, whereas a broadband horn antenna with 24-dBi gain has been applied as a receiver antenna. Finally, an MS2830A signal analyzer from Anritsu has been used to analyze the received spectrum, as reported in Fig. 6. Fig. 6(a) demonstrates the maximum power level of $-45.5 \mathrm{~dB} \cdot \mathrm{m}$ at $28 \mathrm{GHz}$, which has been obtained by using $S c 1$. It was -61.5 and $-50.8 \mathrm{~dB} \cdot \mathrm{m}$, i.e., 16 and $5.3 \mathrm{~dB}$ lower than $S c 1$, for the scenarios $S c 2$ and $S c 3$, respectively. Not only is the antenna gain reduced, but also its main lobe is steered to another angle, as soon as the laser is turned off (optical reconfiguration). By means of realigning the transmitting and receiving antennas (scenario $S c 3$ ), we could avoid the misalignment and conclude the antenna optical control results in a gain enhancement of $5.3 \mathrm{~dB}$. Finally, the power obtained using $S c 4$ was only $-65 \mathrm{~dB} \cdot \mathrm{m}$ because the antenna design had been conducted by taking into consideration the PS electromagnetic properties; therefore, the antenna impedance matching is lost, as soon as the switches are removed.
The power difference between $S c l$ and other scenarios is further increased at $38 \mathrm{GHz}$, as presented in Fig. 6(b), because the beamwidth is narrow for this frequency band. For this reason, the misalignment is even more critical. The power level was $-43 \mathrm{~dB} \cdot \mathrm{m}$ for $S c 1$ and -55.6 and $-48.4 \mathrm{~dB} \cdot \mathrm{m}$ for $S c 2$ and $S c 3$, respectively. Therefore, the gain improvement due to the optical control was $5.4 \mathrm{~dB}$ for this case.

\section{CONCLUSION}

An OCRAA for mm-wave applications has been designed, fabricated, and characterized. The proposed design enables bandwidth management through two different frequency bands, namely: a $10.95 \%$ band centered at $28 \mathrm{GHz}$ and a $4.25 \%$ band at $38 \mathrm{GHz}$. In this manner, we significantly enhance the degrees of freedom in the antenna design, opening new possibilities in the development of reconfigurable antennas for mm-wave frequency range and so on. Furthermore, the antenna radiation pattern was numerically and experimentally characterized, demonstrating a gain of approximately 8.0 and $9.0 \mathrm{dBi}$ for 28 and $38 \mathrm{GHz}$, respectively. The proposed optically controlled antenna has potential for use in mm-wave indoor applications based on optical-wireless networks, in which the optical fibers can be used not only to transmit data, but also to remotely control the antenna electromagnetic properties.

\section{REFERENCES}

[1] S. Koenig et al., "Reconfigurable antennas for wireless and space applications," Proc. IEEE, vol. 100, no. 7, pp. 2250-2261, Jul. 2012

[2] A. C. Sodré, I. F. da Costa, L. T. Manera, and J. A. Diniz, "Optically controlled reconfigurable antenna array based on E-shaped elements," Int. J. Antennas Propag., vol. 2014, 2014, Art. no. 750208.

[3] J. Costantine, Y. Tawk, S. E. Barbin, and C. G. Christodoulou, "Reconfigurable Antennas: Design and Applications," Proc. IEEE, vol. 103, no. 3, pp. 424-437, Mar. 2015.

[4] Y. Tawk, A. R. Albrecht, S. Hemmady, G. Balakrishnan, and C. G. Christodoulou, "Optically pumped frequency reconfigurable antenna design,” IEEE Antennas Wireless Propag. Lett., vol. 9, pp. 280-283, 2010.

[5] Y. Tawk et al., "Demonstration of a cognitive radio front end using an optically pumped reconfigurable antenna system (OPRAS)," IEEE Trans. Antennas Propag., vol. 60, no. 2, pp. 1075-1083, Feb. 2012.

[6] C. J. Panagamuwa, A. Chauraya, and J. C. Vardaxoglou, "Frequency and beam reconfigurable antenna using photoconducting switches," IEEE Trans. Antennas Propag., vol. 54, no. 2, pp. 449-454, Feb. 2006.

[7] E. K. Kowalczuk, R. D. Seager, C. J. Panagamuwa, K. ass, and J. C. Vardaxoglou, "Optimising the performance of an optically controlled microwave switch," in Proc. Loughborough Antennas Propag. Conf., Nov. 2012, pp. 1-5.

[8] J. L. Volakis, Antenna Engineering Handbook, 4th ed. New York, NY, USA: McGraw-Hill, 2007.

[9] I. F. da Costa, A. C. Sodré, L. G. Silva, D. H. Spadoti, and A. Bogoni, "Triband slotted waveguide antenna array for millimetric-waves applications,' in Proc. 8th Eur. Conf. Antennas Propag., Apr. 2014, pp. 2052-2054.

[10] R. S. Elliot and L. A. Kurtz, "The design of small slot arrays," IEEE Trans. Antennas Propag., vol. AP-26, no. 2, pp. 214-219, Mar. 1978.

[11] S. Sekretarov and D. M. Vavriv, "A wideband slotted waveguide antenna array for SAR systems," Prog. Electromagn. Res. M, vol. 11, pp. 165-176, 2010.

[12] C. Kittel, Introduction to Solid State Physics, 7th ed. New York, NY, USA: Wiley, 1996

[13] C. A. Balanis, Advanced Engineering Electromagnetics. New York, NY, USA: Wiley, 1989, pp. 63-81.

[14] A. M. Yadav, C. J. Panagamuwa, and R. D. Seager, "Investigation of a plug hole shaped frequency and pattern reconfigurable antenna using photo-conductive microwave switches," in Proc. 41st Eur. Microw. Conf., Manchester, U.K., 2011, pp. 878-881. 